\title{
Individual-level factors associated with intentions to quit smoking among adult smokers in six cities of China: findings from the ITC China Survey
}

\author{
Guoze Feng, ${ }^{1}$ Yuan Jiang, ${ }^{1}$ Oiang Li, ${ }^{1}$ Hua-Hie Yong, ${ }^{2}$ Tara Elton-Marshall, ${ }^{3}$ \\ Jilan Yang, ${ }^{4}$ Lin Li, ${ }^{2}$ Natalie Sansone, ${ }^{3}$ Geoffrey T Fong ${ }^{3}$
}

${ }^{1}$ Tobacco Control Office, Chinese Center for Disease Control and Prevention, Beijing, China

${ }^{2}$ VicHealth Centre for Tobacco Control, The Cancer Council Victoria, Melbourne, Australia ${ }^{3}$ Department of Psychology, University of Waterloo, Waterloo, Canada

${ }^{4}$ Department of Health Studies and Gerontology, University of Waterloo, Waterloo, Canada

\section{Correspondence to}

Guoze Feng, Tobacco Control Office, Chinese Center for Disease Control and Prevention, 27 Nanwei Road, Beijing 100050, P R China; fengguoze@hotmail.com

Received 31 March 2010 Accepted 12 July 2010

\section{Q) UNLCK:D}

This paper is freely available online under the BMJ Journals unlocked scheme, see http:// tobaccocontrol.bmi.com/site/ about/unlocked.xhtml

\begin{abstract}
Background Over 350 million smokers live in China, and this represents nearly one-third of the smoking population of the world. Smoking cessation is critically needed to help reduce the harms and burden caused by smoking-related diseases. It is therefore important to identify the determinants of quitting and of quit intentions among smokers in China. Such knowledge would have potential to guide future tobacco control policies and programs that could increase quit rates in China.
\end{abstract}

Objective To identify the correlates of intentions to quit smoking among a representative sample of adult smokers in six cities in China.

Methods Data from wave 1 (2006) of the International Tobacco Control (ITC) Policy Evaluation Project China Survey, a face-to-face survey of adult Chinese smokers in six cities: Beijing, Shenyang, Shanghai, Changsha, Guangzhou and Yinchuan, was analysed. Households were sampled using a stratified multistage design. About 800 smokers were surveyed in each selected city (total $n=4815$ ).

Results Past quit attempts, duration of past attempts, Heaviness of Smoking Index (HSI), outcome expectancy of quitting, worry about future health and overall opinion of smoking were found to be independently associated with intentions to quit smoking, but demographic characteristics were not.

Conclusions The determinants of quit intentions among smokers in China are fairly similar to those found among smokers in Western countries, despite the fact that interest in quitting is considerably lower among Chinese smokers. Identifying the determinants of quit intentions provides possibilities for shaping effective policies and programs for increasing quitting among smokers in China.

\section{INTRODUCTION}

Currently, approximately 1.3 billion people smoke worldwide and, consequently, 5.4 million people die from tobacco use each year. Tobacco is now ranked as the world's leading killer, as it is a risk factor for six of the eight leading causes of death worldwide. ${ }^{1}$ Though tobacco use is steadily declining in developed countries, smoking prevalence and cigarette consumption are increasing in developing countries. $^{2}{ }^{3}$ It is projected that tobacco use will kill up to 1 billion people during this century, of which $80 \%$ will be in developing countries. ${ }^{1}$

As one of the largest developing countries, China is home to nearly one-third of the world's smokers: a total of about 350 million. At present, an estimated 1 million deaths from smoking occur in China each year, ${ }^{4}$ and if current smoking rates continue, as many as 100 million people currently under the age of 30 in China will die from tobacco use. Thus, it is clear that tobacco control in China plays a critical role in global tobacco control efforts.

Smoking cessation is a priority for preventing smoking-attributable disease and reducing its burden. ${ }^{6}$ Quitting smoking at any age confers substantial and immediate health benefits, including reduced risks of stroke, cardiovascular disease and smoking-related cancers, ${ }^{6-9}$ and quitting smoking by the age of 30 reduces the risk of dying from tobacco-related diseases by almost 90\%. ${ }^{10}$ The World Bank suggests that if adult cigarette consumption were to decrease by half in the year 2020, approximately 180 million tobaccoattributable deaths could be avoided. ${ }^{2}$ Therefore, promotion of smoking cessation has been proposed as a primary focus of tobacco control efforts, especially in developing countries where smoking prevalence and cigarette consumption are both still relatively high.

According to stage-based models of behaviour change, ${ }^{11}$ individual smokers must progress through several stages of behaviour change in order to quit smoking. They begin with no plan to stop smoking, then form an intention to quit, prepare themselves to quit, enact the new behaviour of quitting and finally maintain this behaviour. Having a quit intention is thus a prerequisite for preparing and taking action. ${ }^{12} 13$ Although having an intention to quit is not the only determinant of successful smoking cessation, it is strongly associated with making quit attempts and smoking cessation. ${ }^{14}{ }^{15}$ In Western countries making quit attempts has been found to be associated with the following sociodemographics: being male, ${ }^{16}$ younger, ${ }^{14}{ }^{17-19}$ well educated, ${ }^{19}$ and of white race. ${ }^{20}$ The other reported correlates of quit attempts include level of nicotine dependence, ${ }^{14} \quad 18 \quad 21-24$ measures of motivation, ${ }^{14} 2425$ self-efficacy, ${ }^{26} 27$ and past quit attempts. ${ }^{14} 1725$ China, as with many other developing countries, has little data on levels and correlates of smokers' intentions to quit. A national study on smoking behaviour conducted in 1996 found that smokers' self-reported health status, health concern, family opinions, as well as education and financial status were associated with their quitting behaviour. ${ }^{28} 29$ These are similar to the recent findings from the National Health Service Surveys. ${ }^{30}$ Two studies conducted among 
medical workers, teachers, factory workers and secondary students in Hong Kong and mainland China found that being male, married and having high quitting self-efficacy were associated with the intention to quit smoking. ${ }^{31} 32$ However, to our knowledge, there are no population-based studies of intentions to quit smoking in China.

The purpose of this study was to examine the associations between intentions to quit smoking and a range of individuallevel predictors, including measures of demographics, quitting history, nicotine dependence and motivation to quit.

\section{METHODS}

\section{Data source}

The International Tobacco Control (ITC) ${ }^{30}$ Policy Evaluation Project China Survey is a prospective cohort survey conducted in six cities in China: Beijing, Shanghai, Guangzhou, Shenyang, Changsha and Yinchuan. (A seventh city (Zhengzhou) was dropped because of poor data quality.) These cities were selected based on geographical representations and levels of economic development. The target population of the ITC China Survey consists of smokers and non-smokers who are 18 years or older and are permanent residents who live in residential buildings in each of the six cities. Smokers are defined as those who have smoked at least 100 cigarettes in their lifetime and are currently smoking at least once a week. Within each city there was a random sample selected using a stratified multistage design, with inclusion probabilities proportional to size at the first few stages in each stratum. In each of the 6 cities, 10 Jie Dao (street districts) were randomly selected at the first stage, with probability of selection proportional to the population size of the Jie Dao. Within each selected Jie Dao, two Ju Wei Hui (residential blocks) were selected, again using probability proportional to the population size of the Ju Wei Hui. Within each selected Ju Wei Hui, a complete list of addresses of the dwelling units (households) was first compiled, and then a sample of 300 households was drawn from the list by simple random sampling without replacement. In the enumeration process, information on age, gender and smoking status for all adults living in these 300 households was collected. The enumerated 300 households were then randomly ordered, and adult smokers and nonsmokers were then approached following the randomised order until 40 adult smokers and 10 non-smokers were surveyed. In each city, 800 smokers and 200 non-smokers were selected, so that the overall sample size of the survey is 4815 adult smokers and 1200 adult non-smokers. However, only smokers were included in the analyses for this paper. The wave 1 survey was conducted from February to April of 2006. The cooperation rates ranged from approximately $80.0 \%$ in Beijing and Guangzhou to $95.0 \%$ in Changsha, and the response rates ranged from $39.4 \%$ in Yinchuan to $66.0 \%$ in Guangzhou. Additional information about the ITC China survey methodology and sampling is provided by $\mathrm{Wu}$ et $a^{33}$ and available at http://www.itcproject.org.

The survey was conducted in Mandarin through face-to-face interviews. After providing the potential respondent with information about the survey and completion of the consent form, the average time to complete the survey was $31.4 \mathrm{~min}$ for smokers. A small gift worth about $¥ 10$ was given to each participant as compensation. All materials and procedures used in the ITC China Survey were reviewed and cleared for ethics by the Research Ethics Boards at the University of Waterloo and The Cancer Council Victoria, and by the Institutional Review Boards at the China National Centers for Disease Control and Prevention.

\section{Dependent variable}

Intentions to quit were based on responses to the question: 'Do you plan to quit smoking?'. Subjects who selected 'in the next month', 'in the next 6 months' or 'sometime in the future after 6 months' were defined as having an intention to quit, and those who responded 'not at all' were defined as having no intention to quit. This measure was dichotomised for two reasons. First, our primary interest was not on the strength of the intention but rather whether people had an interest in quitting or not. Second, the frequency distribution of this measure was highly skewed with the majority being in the no intention category.

\section{Independent variables}

Sociodemographic variables were gender (female, male), age at survey $(18-24=1,25-39=2,40-54=3,55$ and older $=4)$, income (those with monthly household income less than $¥ 1000$ were coded as 'low income', those between $¥ 1000-3000$ were coded as 'medium income' and those equal or greater than $¥ 3000$ were coded as 'high income', those who did not provide an answer to this question were coded as 'don't know'), education ('low education' levels were based on those who reported as either illiterate or having only primary school education, 'medium education' levels were those with high school or technical secondary school education and 'high education' levels were those with university or junior college education).

Motivational variables assessed were outcome expectancy of quitting, worries about health in the future, favourable attitudes towards smoking and overall opinion about smoking. Outcome expectancy of quitting was measured using the question: 'How much do you think you would benefit from health and other gains if you were to quit smoking permanently in the next 6 months?'. Response categories included: 'not at all', 'a little', 'very much' and 'don't know'. Worries about health in the future were measured by asking: 'How worried are you, if at all, that smoking will damage your health in the future?'. Response categories included: 'not at all', 'a little', 'very much' and 'don't know'. Favourable attitude towards smoking was measured by asking for rating of agreement with the statement: 'You enjoy smoking too much to give it up'. The variable was coded into a three-category variable because of small numbers for some categories: disagree (strongly disagree, disagree), neither disagree nor agree and agree (agree, strongly agree). Overall opinion about smoking was measured by asking: 'What is your overall opinion of smoking?'. This variable was also coded as a three-category variable because of small numbers for some categories: good (very good, good), neither good nor bad and bad (bad, very bad)

Quitting history variables assessed were: tried to quit smoking within last year (yes, no) and longest time off smoking (never, less than 1 month, between $1-6$ months, 6 months or more, don't know).

Nicotine dependence was measured using the Heaviness of Smoking Index (HSI), which was based on the sum of two categorical variables: number of cigarettes smoked per day (scored as 0: 0-10 cigarettes per day (CPD), 1: 11-20 CPD, 2: 21-30 CPD, 3: >31 CPD) and time to first cigarette (scored as 0: $>61 \mathrm{~min}, 1$ : $31-60 \mathrm{~min}, 2: 6-30 \mathrm{~min}, 3: 5 \mathrm{~min}$ or less).

\section{Statistical analysis}

For wave 1 data, the weights were simply calculated as the reciprocal of the inclusion probabilities, the final weight for a sampled individual was the number of people in the city population and the sampling category represented by that individual. $^{33}$ Unless otherwise indicated, data analyses were conducted on weighted data using SPSS V.17.0 Complex Survey 
procedures (SPSS, Chicago, Illinois, USA). Logistic regression models were employed to determine the correlates of intentions to quit, first bivariately just between the dependent variable and each of the independent variables of interest, and then multivariately where the association between each independent variable and the dependent variable was adjusted for all other variables in the models. For multivariate analysis, all independent variables were entered into the multivariate logistic regression model to determine their independent effects. An $\alpha$ level of $p<0.05$ was used to determine the level of statistical significance.

\section{RESULTS}

Table 1 shows the sample characteristics by city. Of the 4815 smokers interviewed, 4574 were men $(95.0 \%)$ and 241 women $(5.0 \%)$, with an average age of 50.1 years $(\mathrm{SD}=12.67)$. The 18-24 years age group was under-represented in our sample. The majority (95.0\%) of the respondents were Han Chinese, with other ethnic groups accounting for the rest. Most of the subjects had a medium level of education (high school and technical secondary school, 65.5\%). Almost half of the sample had an average family monthly income of $¥ 1000-3000$ (48.4\%), while those making more than $¥ 3000$ or less than $¥ 1000$ accounted for $30.5 \%$ and $21.1 \%$, respectively. More than half of the smokers surveyed (53.1\%) had attempted to quit smoking before, and almost one-quarter (23.6\%) planned to quit.

Table 2 shows the results for univariate and multivariate analyses. The proportion of smokers with an intention to quit smoking was not significantly different across gender, age groups and income levels. Compared to smokers with a low education level, those with either a medium education level (OR 1.39, 95\%
CI 1.00 to 1.92), or a high education level (OR 1.60, 95\% CI 1.10 to 2.32 ) had significantly greater intentions to quit smoking, but the differences were no longer significant once we controlled for other covariates in the multivariate analysis. Smokers who had made a quit attempt within the last year and also those who had longer time off smoking were more likely to have an intention to quit smoking (significant in the univariate and multivariate analyses). However, smokers who scored higher on the HSI were less likely to have an intention to quit smoking in the univariate and multivariate analyses. Motivational variables, including greater perceived benefits from quitting, worry about the health effects of smoking, negative attitudes towards smoking and low overall opinion of smoking were found to be significantly associated with having an intention to quit in the univariate and multivariate analyses.

\section{DISCUSSION}

One key finding from this study is that the level of interest in quitting among Chinese smokers from the six cities studied was found to be generally low (ranging from 15\% to 31\%) and certainly considerably lower than that reported by Hyland et al in four developed countries in the West that ranged from $65 \%$ to $81 \% .{ }^{34}$ This finding underscores the need for greater effort to be made to stimulate interest in quitting among Chinese smokers in order to help China make significant inroad in reducing the health burden due to tobacco-related diseases.

Despite the low level of interest in quitting, consistent with the findings from Western countries, ${ }^{14} 3536$ intentions to quit smoking among smokers from the six cities in China were found to be influenced by similar individual-level factors such as the HSI, a behavioural measure of nicotine dependence, past quitting behaviour and motivational variables.

Table 1 Sample characteristics by city

\begin{tabular}{|c|c|c|c|c|c|c|c|}
\hline Variables & $\begin{array}{l}\text { Total, } \\
n=4815\end{array}$ & $\begin{array}{l}\text { Beijing, } \\
\mathrm{n}=\mathbf{8 0 4}\end{array}$ & $\begin{array}{l}\text { Shenyang, } \\
\mathrm{n}=\mathbf{8 0 1}\end{array}$ & $\begin{array}{l}\text { Shanghai, } \\
\mathrm{n}=\mathbf{8 0 1}\end{array}$ & $\begin{array}{l}\text { Changsha, } \\
n=803\end{array}$ & $\begin{array}{l}\text { Guangzhou, } \\
\mathbf{n = 8 0 4}\end{array}$ & $\begin{array}{l}\text { Yinchuan, } \\
n=802\end{array}$ \\
\hline \multicolumn{8}{|l|}{ Ethnicity } \\
\hline Han & 95.0 & 94.5 & 94.9 & 98.6 & 98.6 & 99.3 & 84.2 \\
\hline Other & 5.0 & 5.5 & 5.1 & 1.4 & 1.4 & 0.7 & 15.8 \\
\hline \multicolumn{8}{|l|}{ Gender } \\
\hline Male & 95.0 & 94.5 & 97.6 & 91.5 & 94.4 & 97.4 & 94.5 \\
\hline Female & 5.0 & 5.5 & 2.4 & 8.5 & 5.6 & 2.6 & 5.5 \\
\hline \multicolumn{8}{|l|}{ Age } \\
\hline $18-24$ & 1.4 & 2.0 & 1.6 & 1.0 & 1.7 & 0.9 & 1.1 \\
\hline $25-39$ & 17.6 & 13.6 & 12.6 & 10.5 & 23.4 & 14.0 & 31.5 \\
\hline $40-54$ & 49.4 & 47.4 & 57.9 & 57.8 & 45.3 & 44.4 & 43.4 \\
\hline$\geq 55$ & 31.6 & 37.0 & 27.9 & 30.7 & 29.6 & 40.7 & 24.0 \\
\hline \multicolumn{8}{|c|}{ Household income per month* } \\
\hline Low $(<¥ 1000)$ & 19.6 & 9.3 & 31.5 & 14.3 & 28.3 & 12.1 & 22.1 \\
\hline $\begin{array}{l}\text { Medium } \\
(¥ 1000-3000)\end{array}$ & 44.9 & 40.8 & 55.5 & 44.0 & 41.8 & 37.1 & 49.9 \\
\hline High ( $\geq ¥ 3000)$ & 28.3 & 41.6 & 10.0 & 37.8 & 24.7 & 36.2 & 19.5 \\
\hline Don't know & 7.3 & 8.2 & 3.0 & 4.0 & 5.2 & 14.7 & 8.5 \\
\hline \multicolumn{8}{|l|}{ Education } \\
\hline Low & 13.1 & 9.6 & 7.9 & 6.0 & 17.7 & 23.8 & 13.6 \\
\hline Medium & 65.5 & 62.9 & 73.1 & 74.4 & 60.8 & 59.9 & 61.7 \\
\hline High & 21.5 & 27.5 & 19.0 & 19.6 & 21.5 & 16.4 & 24.7 \\
\hline \multicolumn{8}{|c|}{ Ever tried to quit smoking } \\
\hline No & 46.9 & 48.6 & 48.8 & 59.8 & 40.8 & 44.4 & 38.8 \\
\hline Yes & 53.1 & 51.4 & 51.2 & 40.3 & 59.2 & 55.6 & 61.2 \\
\hline \multicolumn{8}{|l|}{ Intention to quit } \\
\hline No & 76.4 & 75.3 & 69.2 & 83.6 & 74.4 & 85.1 & 71.1 \\
\hline Yes & 23.6 & 24.7 & 30.8 & 16.4 & 25.6 & 14.9 & 28.9 \\
\hline
\end{tabular}

* $¥ 1=$ US $\$ 0.1464$ (as of 30 May 2010). Equivalents are low income: <US\$146, medium income: US $\$ 146-439$, high income: $\geq$ US $\$ 439$. 
Table 2 Individual-level factors associated with intentions to quit smoking

\begin{tabular}{|c|c|c|c|c|c|c|}
\hline & \multirow[b]{2}{*}{$\mathbf{n}$} & \multirow[b]{2}{*}{ Intention to quit(\%) } & \multicolumn{2}{|c|}{ Univariate analysis } & \multicolumn{2}{|c|}{ Multivariate analysis } \\
\hline & & & $\overline{\mathbf{O R}}$ & $95 \% \mathrm{Cl}$ & $\overline{\mathbf{O R}}$ & $95 \% \mathrm{CI}$ \\
\hline \multicolumn{7}{|l|}{ Gender } \\
\hline Female & 232 & 25.3 & Reference & & Reference & \\
\hline Male & 4481 & 24.3 & 0.95 & 0.56 to 1.61 & 0.81 & 0.43 to 1.54 \\
\hline \multicolumn{7}{|l|}{ Age } \\
\hline $18-24$ & 66 & 29.6 & Reference & & Reference & \\
\hline $25-39$ & 828 & 25.4 & 0.81 & 0.41 to 1.61 & 0.95 & 0.43 to 2.11 \\
\hline $40-54$ & 2330 & 24.6 & 0.77 & 0.35 to 1.69 & 1.08 & 0.44 to 2.64 \\
\hline$\geq 55$ & 1489 & 23.2 & 0.72 & 0.35 to 1.46 & 0.94 & 0.40 to 2.23 \\
\hline \multicolumn{7}{|l|}{ Household income per month } \\
\hline Low $(<¥ 1000)$ & 923 & 22.8 & Reference & & Reference & \\
\hline Medium ( $¥ 1000-3000)$ & 2125 & 25.6 & 1.17 & 0.92 to 1.47 & 1.13 & 0.87 to 1.47 \\
\hline High $(\geq ¥ 3000)$ & 1323 & 25.5 & 1.16 & 0.87 to 1.54 & 1.29 & 0.94 to 1.77 \\
\hline Don't know & 339 & 16.6 & 0.67 & 0.46 to 0.99 & 0.92 & 0.55 to 1.57 \\
\hline \multicolumn{7}{|l|}{ Education } \\
\hline Low & 619 & 19.1 & Reference & & Reference & \\
\hline Medium & 3085 & 24.7 & 1.39 & 1.00 to 1.92 & 1.21 & 0.84 to 1.74 \\
\hline High & 1004 & 27.3 & 1.60 & 1.10 to 2.32 & 1.29 & 0.83 to 1.98 \\
\hline \multicolumn{7}{|l|}{ Tried to quit within last year } \\
\hline No & 3939 & 18.7 & Reference & & Reference & \\
\hline Yes & 777 & 51.0 & 4.52 & 3.74 to 5.45 & 2.29 & 1.81 to 2.89 \\
\hline \multicolumn{7}{|l|}{ Longest time quit smoking } \\
\hline Never quit & 2211 & 13.2 & Reference & & Reference & \\
\hline Less than 1 month & 1027 & 32.7 & 3.19 & 2.70 to 3.78 & 1.34 & 1.04 to 1.71 \\
\hline $1-6$ months & 893 & 36.8 & 3.84 & 2.66 to 5.53 & 1.91 & 1.39 to 2.61 \\
\hline 6 months or more & 548 & 34.5 & 3.46 & 2.67 to 4.49 & 2.19 & 1.64 to 2.92 \\
\hline Don't know & 30 & 22.3 & 1.89 & 0.78 to 4.56 & 1.90 & 0.66 to 5.49 \\
\hline \multicolumn{7}{|l|}{$\mathrm{HSI}$} \\
\hline $0-6$ & 4381 & & 0.83 & 0.79 to 0.88 & 0.87 & 0.82 to 0.93 \\
\hline \multicolumn{7}{|c|}{ Outcome expectancy of quitting (benefit from quitting in the next 6 months) } \\
\hline Not at all & 942 & 7.3 & Reference & & Reference & \\
\hline A little & 1523 & 21.0 & 3.37 & 2.25 to 5.05 & 2.14 & 1.41 to 3.25 \\
\hline Very much & 1608 & 43.3 & 9.71 & 6.33 to 14.87 & 3.84 & 2.44 to 6.04 \\
\hline Don't know & 638 & 7.7 & 1.06 & 0.65 to 1.73 & 1.01 & 0.60 to 1.70 \\
\hline \multicolumn{7}{|c|}{ Worried about health in the future } \\
\hline Not at all & 1634 & 8.5 & Reference & & Reference & \\
\hline A little & 1976 & 27.3 & 4.05 & 3.04 to 5.40 & 2.04 & 1.58 to 2.62 \\
\hline Very much & 853 & 49.5 & 10.59 & 7.96 to 14.09 & 3.36 & 2.32 to 4.88 \\
\hline Don't know & 249 & 13.6 & 1.70 & 0.99 to 2.92 & 1.54 & 0.85 to 2.76 \\
\hline \multicolumn{7}{|c|}{ Favourable attitudes about smoking } \\
\hline Neither disagree nor agree & 456 & 14.2 & Reference & & Reference & \\
\hline Agree & 2591 & 23.0 & 1.81 & 1.19 to 2.75 & 2.01 & 1.08 to 3.74 \\
\hline Disagree & 1584 & 30.0 & 2.60 & 1.77 to 3.81 & 2.09 & 1.18 to 3.71 \\
\hline \multicolumn{7}{|l|}{ Overall opinion of smoking } \\
\hline Neither good nor bad & 1800 & 13.6 & Reference & & Reference & \\
\hline Bad & 2480 & 34.9 & 3.42 & 2.87 to 4.07 & 1.70 & 1.33 to 2.16 \\
\hline Good & 315 & 6.4 & 0.44 & 0.27 to 0.72 & 0.66 & 0.40 to 1.09 \\
\hline
\end{tabular}

Bold values indicate a significant difference at $p<0.05$.

HSI, Heaviness of Smoking Index.

The majority of previous studies have shown that demographic characteristics such as gender, age, income and education are associated with making serious quit attempts and smoking cessation, ${ }^{15}$ 17-19 37-39 $^{37}$ but the relation between demographic characteristics and intentions to quit smoking are not always consistent. ${ }^{29} 364041$ In our study, quitting intention was not independently associated with age, income and education levels. The lack of an independent effect for education might be because it was confounded with other variables in the model, such as the HSI, which has been shown previously to be associated with socioeconomic status. ${ }^{42}$ The lack of demographic differences in quit intentions might also reflect cultural differences, ${ }^{41}$ a possibility that awaits further research. A recently published study which used data from China National Health Service Surveys conducted in 1993, 1998 and 2003 found that quitting increased with age among Chinese smokers, and a considerable proportion of former smokers (40.6\%) quit because of illness. ${ }^{30}$ As mentioned earlier, a cross-sectional study among Hong Kong Chinese smokers conducted by Abdullah and Yam found that being male was associated with intention to quit, ${ }^{31}$ but this was not found in our study. One possible reason for this difference is the small sample size of our study, especially for women smokers (Abdullah and Yam's study had a much bigger sample size; 11700 persons were included). Another possible explanation for the difference in predictors of intention to quit between the Hong Kong and mainland Chinese smokers 
could be the difference in tobacco control culture in Hong Kong versus mainland China. Compared to mainland China, Hong Kong has a much longer history in tobacco control. It has had well implemented smoke-free policies in workplaces and public venues, and much more substantial anti-smoking campaigns and cessation services. Compared to smokers in this study, the Hong Kong Chinese smokers had a much higher rate of intention to quit; of the daily smokers, $52 \%$ intended to quit. ${ }^{31}$

Previous research in the West has found that nicotine dependence is a significant barrier to making quit attempts and smoking cessation. ${ }^{14} 15{ }^{36}$ Using intentions to quit as an outcome variable, we found a similar negative relation, that is, the higher the level of nicotine dependence, the lower the willingness to quit. This finding suggests that cessation strategies should be tailored to the smoker's level of nicotine dependence. Smokers with low dependence should be encouraged to make quit attempt. Smokers with higher nicotine dependence should be treated with interventions that help to reduce consumption in order to increase their chances of being able to quit successfully in the future. ${ }^{43-45}$

Consistent with the findings from Western countries showing that past quitting experiences are associated with subsequent quitting attempts, ${ }^{14} 35$ we found the same factor predicted intentions to quit smoking. This suggests that smokers without a quit history may have no intention to quit, and thus brief interventions can be designed to stimulate motivation to quit smoking among this group. Brief interventions involve opportunistic advice, discussion, negotiation or encouragement. They are often delivered by a range of primary and community care professionals. For smoking cessation, brief interventions typically take between $5-10$ min. ${ }^{46}$ Given that successful quitting requires repeated interventions and multiple attempts to quit, ${ }^{47}$ professional counselling and medical treatments should also be provided to increase the chances of successful quitting. However, cessation services and quitting medications (eg, nicotine replacement therapy medications) are not generally available in China, especially in rural areas. Efforts need to be made to train doctors and health professionals in providing brief cessation interventions or making referrals to cessation services. ${ }^{30} 48$

Our finding of an independent effect of motivational variables on quit intentions is consistent with the evidence from other studies including national surveys in China, which show that major reasons for quitting include present illness ${ }^{29} 49$ and future health concerns. ${ }^{50}$ Misconceptions and lack of awareness of health risks are common among Chinese smokers, ${ }^{52}$ so public education campaigns are needed to increase smokers' awareness of the health harms of smoking, which in turn can increase their interest in quitting.

Smokers' intentions to quit smoking are also clearly influenced by their attitudes towards smoking. Smoking is common in China, especially among men. Many regard smoking and exchanging cigarettes as a normal part of life. ${ }^{53}$ Consequently, there is a critical need to change the social acceptability of smoking in order to change the attitudes of smokers towards smoking. Health warnings on cigarette packaging are one of the most cost-effective approaches in communicating the harms of smoking to smokers, and where pictorial warnings are used, they will have even stronger effects, especially among those with low literacy. ${ }^{1}$ However, China currently uses only text-based warnings that have been proven to be ineffective, so the implementation of pictorial warnings should be advocated.

This study has some limitations. One limitation is the use of respondent reports to provide information, which may be subject to recall bias and social desirability. Furthermore, survey

\section{What this paper adds}

Interest in quitting is considerably lower among Chinese smokers.

- Individual-level factors such as past quit attempts, duration of past attempts, Heaviness of Smoking Index (HSI), outcome expectancy of quitting, worry about future health and overall opinion of smoking were found to be independently associated with intentions to quit smoking.

- Demographic characteristics were not associated with intentions to quit smoking.

response rates in the first wave were moderate to low, therefore, the findings are limited by potential non-response differentials. Socioeconomic variables were relatively difficult to measure (as a construct and its relationship to health and disease factors), and certain measures in our data (ie, income) had a large proportion of 'unknown' responses, which potentially limited the findings. In addition, our survey was limited to urban areas (ie, six selected cities) that are inhabited mainly by the Han ethnic Chinese. In reality, the vast majority of the Chinese smoking population still live in rural areas, where they have a higher smoking prevalence. ${ }^{3049}$ Therefore, caution needs to be exercised to generalise the findings to rural areas and/or minority ethnic groups.

Data analysed in this paper are from the first wave of the ITC China survey, and so causal directionality is somewhat unclear. With the subsequent waves of the data, we will be able to examine prospectively the predictors of making quit attempts. In addition to individual factors, social environmental factors may also be important drivers of quitting and these will be examined in future papers. It is reassuring that the findings from this study are generally consistent with those from the Western countries despite the linguistic and/or cultural differences which could potentially affect the interpretation of survey questions, including factors associated with intentions to quit.

In summary, like their Western counterparts, interest in quitting among smokers from six cities in China is influenced by similar individual-level factors such as past quitting experiences, nicotine dependence, health concerns and their attitudes towards smoking, underscoring the need to consider these factors when designing cessation intervention programs to ensure that they are effective.

Acknowledgements The authors would like to acknowledge the Chinese Center for Disease Control and Prevention and local CDC representatives in each city for their role in data collection.

Funding The ITC China Project was supported by grants from the US National Cancer Institute (R01 CA125116 and the Roswell Park Transdisciplinary Tobacco Use Research Center (P50 CA111236)), Canadian Institutes of Health Research (79551), Chinese Center for Disease Control and Prevention, and the Ontario Institute for Cancer Research. The funding sources had no role in the study design, in collection, analysis, and interpretation of data, in the writing of the report, or in the decision to submit the paper for publication.

\section{Competing interests None.}

\section{Patient consent Obtained.}

Ethics approval This study was conducted with the approval of the Ethics approval was obtained from the Office of Research Ethics at the University of Waterloo (Waterloo, Canada) and the internal review boards at: Roswell Park Cancer Institute (Buffalo, USA), the Cancer Council Victoria (Melbourne, Australia) and the Chinese Center for Disease Control and Prevention (Beijing, China).

Contributors All authors made significant contributions to different versions of the manuscript. 
Provenance and peer review Not commissioned; externally peer reviewed.

\section{REFERENCES}

1. World Health Organization. WHO Report on the Global Tobacco Epidemic, 2008The MPOWER package, Geneva, 2008.

2. Anon. Curbing the epidemic: governments and the economics of tobacco control. The World Bank. Tob Control 1999;8:196-201.

3. Ezzati M, Lopez AD. Estimates of global mortality attributable to smoking in 2000. Lancet 2003;362:847-52.

4. Lin HH, Murray M, Cohen $\mathrm{T}$, et al. Effects of smoking and solid-fuel use on COPD, lung cancer, and tuberculosis in China: a time-based, multiple risk factor, modelling study. Lancet 2008;372:1473-83.

5. Coffield AB, Maciosek MV, McGinnis JM, et al. Priorities among recommended clinical preventive services. Am J Prev Med 2001:21:1-9.

6. The Surgeon General's 1990 Report on The Health Benefits of Smoking Cessation Executive Summary. MMWR Recomm Rep 1990;39:i-xv, 1-12.

7. Terres $\mathbf{W}$, Becker $P$, Rosenberg $A$. Changes in cardiovascular risk profile during the cessation of smoking. Am J Med 1994;97:242-9.

8. Godtfredsen NS, Lam TH, Hansel TT, et al. COPD-related morbidity and mortality after smoking cessation: status of the evidence. Eur Respir $\mathrm{J}$ 2008;32:844-53.

9. Lam TH, Li ZB, Ho SY, et al. Smoking, quitting and mortality in an elderly cohort of 56,000 Hong Kong Chinese. Tob Control 2007;16:182-9.

10. Doll R, Peto R, Boreham J, et al. Mortality from cancer in relation to smoking: 50 years observations on British doctors. Br J Cancer 2005:92:426-9.

11. Prochaska J0, DiClemente CC, Norcross JC. In search of how people change. Applications to addictive behaviors. Am Psychol 1992:47:1102-14.

12. DiClemente CC, Prochaska J0, Fairhurst SK, et al. The process of smoking cessation: an analysis of precontemplation, contemplation, and preparation stages of change. J Consult Clin Psychol 1991:59:295-304.

13. Prochaska J0, Goldstein MG. Process of smoking cessation. Implications for clinicians. Clin Chest Med 1991;12:727-35.

14. Hyland A, Borland R, Li Q, et al. Individual-level predictors of cessation behaviours among participants in the International Tobacco Control (ITC) Four Country Survey. Tob Control 2006;15(Suppl 3):iii83-94.

15. Hyland A, Li Q, Bauer JE, et al. Predictors of cessation in a cohort of current and former smokers followed over 13 years. Nicotine Tob Res 2004;6(Suppl 3): S363-9.

16. Nides MA, Rakos RF, Gonzales D, et al. Predictors of initial smoking cessation and relapse through the first 2 years of the Lung Health Study. J Consult Clin Psychol 1995:63(1):60-9.

17. Venters MH, Kottke TE, Solberg LI, et al. Dependency, social factors, and the smoking cessation process: the doctors helping smokers study. Am J Prev Med 1990:6:185-93.

18. Vanasse A, Niyonsenga T, Courteau J. Smoking cessation within the context of family medicine: which smokers take action? Prev Med 2004;38:330-7.

19. Hatziandreu EJ, Pierce JP, Lefkopoulou M, et al. Quitting smoking in the United States in 1986. J Natl Cancer Inst 1990;82:1402-6.

20. Tucker JS, Ellickson PL, Orlando $\mathrm{M}$, et al. Predictors of attempted quitting and cessation among young adult smokers. Prev Med 2005;41:554-61.

21. Borland R, Owen N, Hill D, et al. Predicting attempts and sustained cessation of smoking after the introduction of workplace smoking bans. Health Psychol 1991;10:336-42

22. Hellman R, Cummings $\mathrm{K}$, Haughey $\mathrm{B}$, et al. Predictors of attempting and succeeding at smoking cessation. Health Educ Res 1991;6:77-86.

23. Zimmermann R, Warheit G, Ulbrich P. The relationship between alcohol use and attempts and success at smoking cessation. Addict Behav 1990:15:197-207.

24. Clark MA, Kviz FJ, Crittenden KS, et al. Psychosocial factors and smoking cessation behaviors among smokers who have and have not ever tried to quit. Health Educ Res 1998:13:145-53.

25. Burt RD, Peterson AV Jr. Smoking cessation among high school seniors. Prev Med 1998;27:319-27

26. Dijkstra A, de Vries $\mathrm{H}$, Bakker M. Pros and cons of quitting, self-efficacy, and the stages of change in smoking cessation. J Consult Clin Psychol 1996; 64:758-63.
27. Woodruff SI, Conway TL, Edwards CC. Sociodemographic and smoking-related psychosocial predictors of smoking behavior change among high school smokers. Addict Behav 2008;33:354-8.

28. Yang G, Fan L, Tan J, et al. Smoking in China: findings of the 1996 National Prevalence Survey. JAMA 1999;282:1247-53.

29. Yang G, Ma J, Chen A, et al. Smoking cessation in China: findings from the 1996 national prevalence survey. Tob Control 2001;10:170-4.

30. Qian J, Cai M, Gao J, et al. Trends in smoking and quitting in China from 1993 to 2003: National Health Service Survey data. Bulletin of the World Health Organization 2010:19

31. Abdullah AS, Yam HK. Intention to quit smoking, attempts to quit, and successful quitting among Hong Kong Chinese smokers: population prevalence and predictors. Am J Health Promot 2005;19:346-54.

32. Wang SHQ, Borland $\mathrm{R}$, Whelan A. Determinants of intention to quit: Confirmation and extension of western theories in male chinese smokers. Psychology \& Health 2005;20:35-51.

33. Wu C, Thompson ME, Fong GT, et al. Methods of the International Tobacco Control (ITC) China Survey. Tob Control 2010;19(Suppl 2);i1-i5.

34. Hyland A, Laux FL, Higbee C, et al. Cigarette purchase patterns in four countries and the relationship with cessation: findings from the International Tobacco Control (ITC) Four Country Survey. Tob Control 2006;15(Suppl 3):iii59-64

35. Haddad LG, Petro-Nustas W. Predictors of intention to quit smoking among Jordanian university students. Can J Public Health 2006:97:9-13.

36. Fagan $\mathbf{P}$, Augustson $\mathrm{E}$, Backinger $\mathrm{CL}$, et al. Quit attempts and intention to quit cigarette smoking among young adults in the United States. Am J Public Health 2007:97:1412-20.

37. Hymowitz N, Cummings KM, Hyland A, et al. Predictors of smoking cessation in a cohort of adult smokers followed for five years. Tob Control 1997;6(Suppl 2):S57-62.

38. Rose JS, Chassin L, Presson CC, et al. Prospective predictors of quit attempts and smoking cessation in young adults. Health Psychol 1996:15:261-8.

39. Yang T, Abdullah AS, Mustafa J, et al. Factors associated with smoking cessation among Chinese adults in rural China. Am J Health Behav 2009;33:125-34.

40. Droomers M, Schrijvers CT, Mackenbach JP. Educational differences in the intention to stop smoking: explanations based on the Theory of Planned Behaviour. Eur J Public Health 2004:14:194-8.

41. Siahpush $\mathbf{M}$, Borland $\mathrm{R}$, Yong $\mathrm{HH}$, et al. Socio-economic variations in tobacco consumption, intention to quit and self-efficacy to quit among male smokers in Thailand and Malaysia: results from the International Tobacco Control-South-East Asia (ITC-SEA) survey. Addiction 2008;103:502-8.

42. Siahpush M, McNeill A, Borland R, et al. Socioeconomic variations in nicotine dependence, self-efficacy, and intention to quit across four countries: findings from the International Tobacco Control (ITC) Four Country Survey. Tob Control 2006:15(Suppl 3):iiï1-5.

43. Etter JF, Laszlo E, Zellweger JP, et al. Nicotine replacement to reduce cigarette consumption in smokers who are unwilling to quit: a randomized trial. J Clin Psychopharmacol 2002;22:487-95.

44. Hughes JR, Carpenter MJ. The feasibility of smoking reduction: an update. Addiction 2005; 100:1074-89.

45. Hyland A, Levy DT, Rezaishiraz $\mathrm{H}$, et al. Reduction in amount smoked predicts future cessation. Psychol Addict Behav 2005;19:221-5

46. Excellence NIfHaC. Brief interventions and referral for smoking cessation, 2006.

47. Treating Tobacco Use And Dependence: 2008 Update, 2008

48. Jiang Y, Ong MK, Tong EK, et al. Chinese physicians and their smoking knowledge attitudes, and practices. Am J Prev Med 2007;33:15-22.

49. Yang GH, Ma JM, Liv N, et al. Smoking and passive smoking in Chinese, 2002 Chin J Epidemiol 2005;26:77-83

50. Abdullah AS, Ho LM, Kwan YH, et al. Promoting smoking cessation among the elderly: what are the predictors of intention to quit and successful quitting? J Aging Health 2006; 18:552-64

51. Wong DC, Chan SS, Ho SY, et al. Predictors of intention to quit smoking in Hong Kong secondary school children. J Public Health (Oxf) 2009

52. The International Tobacco Control Policy Evaluation Project (ITC) China Summary، 2009.

53. Ma SJ, Wang JF, Mei CZ, et al. Passive smoking in China: contributing factors and areas for future interventions. Biomed Environ Sci 2007:20:420-5. 